\title{
Peripheral Blood Lymphocyte Subsets in Infants with Diarrhea with and without Giardia Lamblia Infection
}

\author{
MICHAEL SCHLESINGER, ESTHER GRANOT, RUTH RABINOWITZ, AND \\ RICHARD J. DECKELBAUM
}

\begin{abstract}
The Hubert H. Humphrey Center for Experimental Medicine and Cancer Research; the Paul Ehrlich Center for the study of White Blood Cells; The Hebrew University-Hadassah Medical School; Department of Pediatrics, the Hadassah University Hospital Jerusalem, Israel; and Department of Pediatrics, College of Physicians and Surgeons of Columbia University, New York, New York 10032
\end{abstract}

\begin{abstract}
The aim of the present study was to define the cellular immune response during gastrointestinal Giardia lamblia infection in young children. The level of lymphocyte subsets was determined in the peripheral blood of infants with $G$. lamblia-associated diarrhea or acute gastroenteritis and from control infants without diarrhea. The proportion of peripheral blood lymphocytes (PBL) expressing the CD8 marker (suppressor cytotoxic $\mathrm{T}$ cells) and the CD57 marker (natural killer cells and subset of CD8+ T cells) was highest in infants with acute gastroenteritis, lower in infants without diarrhea, and lowest among those with $G$. lamblia-associated diarrhea. The level of CD4+ PBL (helper $T$ cells) did not differ significantly among the three groups of children tested. The level of memory, or helper-inducer, CD4+CD29+ PBL was increased markedly in acute gastroenteritis as compared with their level among the other two groups, whereas naive or virgin CD4+CD45RA+ PBL had the reciprocal distribution among the three groups of infants. In contrast to acute gastroenteritis from other causes, G. lamblia-associated diarrhea did not elicit changes in lymphocyte subsets. (Pediatr Res 33: 15-18, 1993)
\end{abstract}

\section{Abbreviations}

NK, natural killer

PBL, peripheral blood lymphocyte

PE, phycoerythrin

Giardia lamblia is an intestinal parasite implicated as a cause of disease ranging from acute gastroenteritis to chronic diarrhea, malabsorption, and failure to thrive (1). Still, most individuals infected with Giardia are apparently asymptomatic. Children with asymptomatic Giardia infection show no disadvantage in terms of growth achievement and may even tend to suffer less from gastrointestinal and respiratory illnesses compared with uninfected children (2). The marked susceptibility of patients with immune deficiency states to chronic and recurrent giardiasis suggests that immune mechanisms play a predominant role in determining the pathogenicity of $G$. lamblia $(3,4)$. Studies on specific anti-Giardia Ig levels during Giardia infection have,

Received March 26, 1992; accepted September 1, 1992.

Correspondence and reprint requests: Prof. Michael Schlesinger, The Hubert H. Humphrey Center for Experimental Medicine and Cancer Research, The Hebrew University Hadassah Medical School, Jerusalem, 91010, Israel.

Supported by grants from the National Institutes of Health (PO1 AI 26497) and from the Goldhirsch Foundation. however, yielded inconclusive results. Whereas in some studies of acute giardiasis an anti-Giardia IgM response was noted (5, $6)$, in others, an increase in specific IgA but not IgM or IgG antibodies was observed (7). Diminished cellular cytotoxicity for $G$. lamblia has been observed in a patient with symptomatic giardiasis resistant to drug therapy (8). Other studies have failed to detect significant differences in the proportion of $\mathrm{B}$ and $\mathrm{T}$ lymphocytes in patients with acute giardiasis, persistent giardiasis, asymptomatic carriers, and controls (5).

Distinct lymphocyte subsets can be identified by their reactivity with MAb $(9,10)$. Helper T lymphocytes can be detected by their reactivity with $\mathrm{CD} 4 \mathrm{MAb}$, whereas suppressor cytotoxic $\mathrm{T}$ lymphocytes can be identified by their reactivity with $\mathrm{MAb}$ against CD8. The CD57 marker (Leu7) is characteristic for human NK cells (11) and for a subset of suppressor-cytotoxic T cells (12). It has recently been shown that CD4+ $\mathrm{T}$ cells can be further subdivided into two subsets-that of the CD29+CD4+ "memory" or "primed" subset and that of the CD45RA+CD4+ subset of "naive" cells $(13,14)$. The level of memory CD4+ T cells is low among infants and young children and increases with age (15).

In the present study, MAb were used to define the cellular immune response during $G$. lamblia-associated diarrhea in infants. The levels of various subsets of lymphocytes were determined in the peripheral blood of infants suffering from acute diarrhea associated with $G$. lamblia infection. In parallel, PBL from infants with acute gastroenteritis and control infants without diarrhea were tested.

\section{MATERIALS AND METHODS}

Subjects studied. The children included in the study, all below the age of $3 y$, were seen at a pediatric outpatient clinic in a rural area during a 12-mo period. Each child was weighed unclothed and measured during the visit at the clinic. The weight and height of the children were expressed as the percentage of the 50th percentile for weight or height for age.

During the study period, 421 children with acute diarrhea were examined in the clinic. Acute diarrhea was defined as the passage of two or more abnormally formed stools per $24 \mathrm{~h}$ for a period of less than $14 \mathrm{~d}$. The stools of all infants who presented with acute diarrhea were examined for trophozoites or cysts of $G$. lamblia. Wet mounts of each stool sample were prepared in $0.9 \%$ $\mathrm{NaCl}$ and examined by an experienced parasitology technician by light microscopy for $10 \mathrm{~min}$. As stool samples were examined within 2 to $5 \mathrm{~h}$ of being obtained, preservatives were not used.

In 19 children $(4.5 \%) G$. lamblia was found in the stools Blood specimens were available for testing from eight of these children. Twelve children with diarrhea but without $G$. lamblia 
were also included for comparison. The stools of all infants with diarrhea were examined for parasites other than $G$. lamblia, including cryptosporidium, and for bacterial pathogens (pathogenic Escherichia coli, Shigella, Salmonella, and Campylobacter). The stools were also assayed by ELISA for the presence of rotavirus using a commercially available diagnostic kit (Eldan Technologies Co., Jerusalem, Israel).

A volume of 0.5 to $1.0 \mathrm{~mL}$ of blood was drawn and assayed for $\mathrm{Hb}$, hematocrit, white blood cell count, differential, and fluorimetric lymphocyte subpopulation studies. The total lymphocyte number per $\mathrm{mm}^{3}$ was calculated by multiplying the total white blood cell number per $\mathrm{mm}^{3}$ by the proportion of lymphocytes.

This study was approved by the medical ethical committee of the Hadassah University Hospital. Informed consent was obtained from the parents of all children studied.

Detailed analysis was carried out with PBL obtained from the following groups of age-matched infants (Table 1): 1) Eight infants with acute diarrhea and $G$. lamblia in their stools; 2) twelve infants (who were not infected with $G$. lamblia) with acute diarrhea; and 3) nine infants, some of whom suffered from mild upper respiratory infections without diarrhea.

Immunofluorescent assays. Venous blood from children was collected in heparin and analyzed by the whole blood method, in which fluorescent MAb were added to the unseparated blood and the immunofluorescence of the lymphocytes was determined after lysis of the red blood cells (16).

The conjugated MAb used were as follows: FITC-conjugated Leu-3 (CD4), PE-conjugated Leu-2 (CD8), and FITC-conjugated Leu-7 (CD57) (all from Becton-Dickinson, Mountain View, CA). PE-labeled 4B4 (CD29) and PE-labeled 2H4 (CD45RA) were obtained from Coulter Immunology, Hialeah, FL.

Fluorescent MAb were added to $100 \mu \mathrm{L}$ of whole blood. The following pairs of MAb were used for two-color flow cytometry: 1) PE-Leu-2:FITC-Leu-3; 2) PE-Leu-2:FITC-Leu-7; 3) PE4B4:FITC-Leu-3; and 4) PE-2H4:FITC-Leu-3.

To $100 \mu \mathrm{L}$ heparinized blood, $5-10 \mu \mathrm{L}$ of each of the two fluorescent $\mathrm{MAb}$ were added. The mixture of blood and antibodies was kept at room temperature for $30 \mathrm{~min}$, and then the erythrocytes were lysed with $2 \mathrm{~mL}$ of a buffered solution of $0.829 \% \mathrm{NH}_{4} \mathrm{Cl}$, as described by Hoffman et al. (16). After two incubations of the blood with $\mathrm{NH}_{4} \mathrm{Cl}$ for $10 \mathrm{~min}$ at room temperature, the suspension was centrifuged at $200 \times g$ for $5 \mathrm{~min}$, and the pellet was washed twice with PBS. Finally, the cells in the washed pellet were resuspended in $0.1 \%$ paraformaldehyde and kept at $4^{\circ} \mathrm{C}$ until they were analyzed by flow cytometry. A FACScan flow cytometer (Becton-Dickinson) was used. The acquisition and analysis of the fluorimetric data were carried out using the Consort 30 program. For each test, at least 10000 cells were acquired. The total lymphocyte population was gated by the usual procedures according to their typical forward light scatter and right-angle light scatter (17). The immunofluorescent staining was determined by two-color flow cytometry using the contour graph program of Consort 30.

Statistical analysis. Data were analyzed by $t$ test.

\section{RESULTS}

Clinical observations. Of the 12 children in the present study with $G$. lamblia-negative acute diarrhea, pathogenic $E$. coli $(0127,0142,0119,0126)$ was cultured in four children and rotavirus was found in two. Among the eight children with Giardia infection one was concomitantly infected with $E$. coli 0119, and in another child, the dwarf tape worm Hymenolepis nana was detected. In the control group without diarrhea, one child was found to have $H$. nana in the stool, and no other pathogens were detected.

The weight and height of the children in the present study were expressed as a percentage of the 50th percentile for age. The weight of children with non-Giardia diarrhea was significantly lower than that of children with Giardia-positive diarrhea $(p<0.05)$ and nonsignificantly lower than that of children without diarrhea (Table 1). The height of children with diarrhea was not significantly different from that of children in the other groups.

The total lymphocyte number per $\mathrm{mm}^{3}$ was lowest in the group with acute diarrhea, highest in the group without diarrhea, and intermediate in the group of infants with Giardia-associated diarrhea (Table 1).

Lymphocyte subsets. Table 2 shows differences among the three groups of infants in the proportion of CD4+ (helper $\mathrm{T}$ cells), CD8+ (suppressor/cytotoxic T cells), and CD57+ (NK cells and a subset of CD8 cells). The proportion of CD4+ lymphocytes (Table 2) and their absolute number (not shown) were higher among infants with $G$. lamblia infection than among control infants without diarrhea and lowest among infants with non-Giardia diarrhea. However, none of these differences was statistically significant.

The population of CD8+ cells in each group showed a pattern opposite to that of the distribution of $\mathrm{CD} 4+$ cells. The proportion of $\mathrm{CD} 8+$ cells was highest among infants with non-Giardia diarrhea, lower in control infants without diarrhea, and lowest among infants with $G$. lamblia infection. The proportion of CD8 + T cells was significantly higher among infants with nonGiardia diarrhea than among infants with $G$. lamblia infection $(p<0.05)$. The children with non-Giardia-associated diarrhea showed a decrease in CD4+ helper $\mathrm{T}$ cells with a concomitant increase in CD8+ suppressor-cytotoxic cells. Thus, the mean CD4:CD8 ratio was lower in this group of infants than in both the Giardia-associated diarrhea $(p<0.05)$ and the control group. It is noteworthy that in infants with $G$. lamblia-associated diarrhea, the CD4:CD8 ratio was in fact in the normal range. Similar to the population of CD8+ cells, the proportion of CD57+ (Leu$7+$ ) cells was highest among infants with acute gastroenteritis and lowest among infants with $G$. lamblia-associated diarrhea, although these differences were not statistically significant (Table 2).

Subsets of $C D 4+$ lymphocytes. The most striking observation in the present study was the finding of a substantial difference in the level of subsets of CD4+ lymphocytes among the three groups of children studied. The proportion of memory-helper $T$ cells, CD4+CD29+ double-positive lymphocytes, and their percentage

Table 1. Clinical data concerning infants studied*

\begin{tabular}{lccc}
\hline \multicolumn{1}{c}{ Clinical parameter } & $\begin{array}{c}\text { Infants with } \\
\text { non-Giardia } \\
\text { diarrhea }\end{array}$ & $\begin{array}{c}\text { Infants without } \\
\text { diarrhea }\end{array}$ & $\begin{array}{c}\text { Infants with Giardia- } \\
\text { associated diarrhea }\end{array}$ \\
\hline No. of infants & 12 & 8 & 8 \\
Age (mo) & $13.5 \pm 6.9$ & $14.5 \pm 5.1$ & $16.4 \pm 6.2$ \\
Weight (\% of 50th percentile) & $90.7 \pm 9.7$ & $99.4 \pm 11.0$ & $100.5 \pm 7.6 \dagger$ \\
Height (\% of 50th percentile) & $97.7 \pm 3.5$ & $100.3 \pm 2.0$ & $100.3 \pm 2.3$ \\
Duration of diarrhea (d) & $4.6 \pm 3.8$ & $5.5 \pm 4.5$ & $3465 \pm 1442$ \\
No. of lymphocytes per $\mathrm{mm}^{3}$ & $2982 \pm 818$ & $4870 \pm 2679$ & \\
\hline
\end{tabular}

$*$ Values are mean $\pm \mathrm{SD}$.

$\dagger$ Weight was significantly higher in infants with Giardia-associated diarrhea than in infants with non-Giardia diarrhea $(p<0.05)$. 
Table 2. T lymphocytes and NK cells among various groups of infants*

\begin{tabular}{lccc}
\hline Lymphocyte subset & $\begin{array}{c}\text { Infants with non- } \\
\text { Giardia diarrhea }\end{array}$ & $\begin{array}{c}\text { Infants without } \\
\text { diarrhea }\end{array}$ & $\begin{array}{c}\text { Infants with Giardia- } \\
\text { associated diarrhea }\end{array}$ \\
\hline CD4+ & $36.05 \pm 6.82(12)$ & $40.39 \pm 7.77(9)$ & $41.55 \pm 7.92(8)$ \\
CD8+ & $29.92 \pm 7.36(11)$ & $23.58 \pm 9.33(9)$ & $21.66 \pm 5.43(8) \dagger$ \\
CD4/CD8 ratio & $1.33 \pm 0.43(11)$ & $1.56 \pm 0.45(9)$ & $1.98 \pm 0.42(8) \dagger$ \\
CD57+ (Leu-7+) & $16.05 \pm 9.92(11)$ & $10.11 \pm 9.15(9)$ & $8.81 \pm 5.88(8)$ \\
\hline
\end{tabular}

$*$ Values are mean \pm SD. Numbers in parentheses are the number of infants tested.

† The proportion of $\mathrm{CD} 8+\mathrm{PBL}$ and the $\mathrm{CD} 4 \mathrm{CD} 8$ ratio in infants with Giardia-associated diarrhea were significantly different from those in infants with non-Giardia diarrhea $(p<0.05)$.

of the total population of helper T cells (CD4+ cells) was $1 \frac{112-}{2}$ to 2 -fold higher among infants with non-Giardia diarrhea as compared with their proportion among either infants without diarrhea or infants with $G$. lamblia infection (Table 3). Although the total number of $\mathrm{CD} 4+$ lymphocytes was lowest in infants with acute non-Giardia diarrhea, the total number of cells of the CD29+CD4+ subset $\left(615 \pm 61\right.$ cells per $\left.\mathrm{mm}^{3}\right)$ was found to be significantly higher than that among infants with Giardia-associated diarrhea $\left(332 \pm 54\right.$ cells per $\left.\mathrm{mm}^{3}\right)(p<0.02)$. The absolute number of CD29+CD4+ lymphocytes was intermediate in the group of infants without diarrhea $\left(476 \pm 126 \mathrm{cells} / \mathrm{mm}^{3}\right)$.

The distribution of naive helper $T$ cells coexpressing the CD45RA and CD4 markers, and their proportion of the total CD4+ population, showed an inverse relationship to that of the memory helper $\mathrm{T}$ lymphocyte subset among the various groups of infants. The level of naive helper $T$ cells was significantly lower among infants with non-Giardia diarrhea than among infants without diarrhea $(p<0.05)$ or infants with $G$. lamblia infection $(p<0.05)$. Thus, in infants with non-Giardia-associated diarrhea, the decrease in helper $T$ cells is due to a decrease only in the level of naive T-helper cells, whereas the level of memory or primed T-helper cells was significantly increased.

\section{DISCUSSION}

MAb to distinct differentiation antigens on human lymphocytes allow discriminating analysis of changes in lymphocyte subsets $(9,10)$. In addition to the identification of $\mathrm{B}$ cells, total $\mathrm{T}$ cells, helper $\mathrm{T}$ cells, suppressor-cytotoxic $\mathrm{T}$ cells, and NK cells, it has become possible to identify subsets within some of these populations of lymphocytes. The population of $\mathrm{CD} 4+$ helper $\mathrm{T}$ cells can now be further divided into two distinct subsets. One CD4+ subset, characterized by the coexpression of the CD45RA marker, originally designated as the suppressor-inducer subset (18) was recently shown to constitute the subset of naive or virgin CD4+ T cells $(13,14)$. The other subset of CD4+ cells, characterized by the coexpression of the C29 marker, was originally considered the helper-inducer subset (19) but was later shown to constitute the population of memory and activated CD4+ T cells $(10,11,20)$. The relative proportion of the two CD4+ T cell subsets changes with age. In cord blood (20) and among PBL of infants, the proportion of CD29+CD4+ cells is low, whereas that of the CD45RA+CD4+ subset is high (15). In vitro activa- tion by various mitogens can result in a transition of naive $T$ cells to primed or memory $T$ cells with a concomitant switch of their cell surface antigenic profile (20). It was, therefore, of interest to determine whether similar dynamic changes can occur in vivo during an acute infectious episode.

The present study demonstrates that gastrointestinal infection in children under the age of $3 \mathrm{y}$ is indeed associated with significant changes in lymphocyte subset composition. In infants with acute diarrhea not associated with $G$. lamblia infection, the proportion of suppressor-cytotoxic lymphocytes (CD8+ cells) was increased with a significant concomitant decrease of the CD4:CD8 ratio. The level of CD 57+ lymphocytes, characteristic for NK cells and for a subset of CD8+ T lymphocytes, was also increased in infants with acute gastroenteritis. Increases in the proportion of CD8+ $(21)$ and Leu-7+ $($ CD $57+)(12,22)$ lymphocytes have previously been found to be associated with various viral infections. In contrast to these marked changes, in infants infected with $G$. lamblia, the level of CD4+, CD8+, and CD57+ lymphocytes and the $\mathrm{CD} 4: \mathrm{CD} 8$ ratio were normal. Normal values were also found in infants without diarrhea, some of whom had mild upper respiratory infections. Additionally, the level of primed or memory CD4+ cells (CD29+CD4 ${ }^{+}$cells), which is low among healthy infants, was strikingly increased in infants with acute gastroenteritis. In parallel, the subset of naive CD4+ T cells (CD45RA+CD4+ cells) was markedly decreased. Not only was the relative proportion of primed CD4+ cells increased in infants with diarrhea, but also their absolute number. This is particularly striking in view of the fact that the absolute number of CD4+ lymphocytes was decreased in these infants. Because the age of the children in the three groups was similar (Table 1), the difference in the level of subsets of CD4+ lymphocytes among them could not be attributed to age differences. Thus, non-Giardia-associated infectious diarrhea constitutes a potent antigenic stimulus responsible for a marked increase in the primed or memory $\mathrm{T}$-helper cell subpopulation. Follow-up studies are required to determine whether these changes are transient or whether infectious diarrhea in children imparts a permanent adult-like subset pattern.

Because nutrition regulates cellular immune responsiveness $(23,24)$, the question arises whether the above-mentioned observations can be attributed to differences in the nutritional status of the three groups of infants. The mean weight of infants with non-Giardia-associated diarrhea was lower than that of the other

Table 3. Lymphocyte subsets among various groups of infants*

\begin{tabular}{lccc}
\hline Lymphocyte phenotype & $\begin{array}{c}\text { Infants with non- } \\
\text { Giardia diarrhea }\end{array}$ & $\begin{array}{c}\text { Infants without } \\
\text { diarrhea }\end{array}$ & $\begin{array}{c}\text { Infants with Giardia- } \\
\text { associated diarrhea }\end{array}$ \\
\hline CD4+CD29+ $\dagger$ & $21.10 \pm 5.37(12)$ & $13.47 \pm 9.51(9) \ddagger$ & $11.47 \pm 6.85(7) \S$ \\
CD4+CD29+/CD4\| & $58.53 \pm 4.05(12)$ & $33.87 \pm 23.07(9) \S$ & $26.33 \pm 11.88(7) \uparrow$ \\
CD4+CD45RA+† & $9.76 \pm 3.88(11)$ & $22.52 \pm 15.53(8) \ddagger$ & $17.58 \pm 9.25(8) \ddagger$ \\
CD4+CD45RA+/CD4\| & $24.59 \pm 7.50(11)$ & $47.09 \pm 23.42(8) \ddagger$ & $38.91 \pm 20.25(8)$ \\
\hline
\end{tabular}

\footnotetext{
$\dagger$ Proportion of PBL coexpressing the two antigenic markers.

$\ddagger p<0.05$ vs infants with non-Giardia diarrhea.

$\S p<0.01 v s$ infants with non-Giardia diarrhea.

$\|$ Proportion of CD4+ PBL coexpressing two antigenic markers.

I $p<0.001$ vs infants with non-Giardia diarrhea.
}

* Values are mean \pm SD. Numbers in parentheses are the number of infants tested. 
infants studied. None of the children suffered from clinically overt dehydration, but as the infants' weights upon recovery were not available, the differences in weight could partly reflect mild dehydration. The lower weight of infants with non-Giardia diarrhea could also reflect some degree of malnutrition. In children over the age of $36 \mathrm{mo}$, malnutrition has been shown to result in impaired immune function as assessed by skin test reactivity (24). No such correlation was, however, found among children 12-35 mo of age (24). Skin tests to commonly encountered recall-antigens evaluate memory immune responsiveness and are dependent on memory $T$ cells. Our study indicates that children with infectious diarrhea exhibit two seemingly conflicting processes-a decrease in the absolute number of CD4+ cells, paralleled by an increase in the absolute number of primed CD4+ cells. Thus, despite the lower mean weight of this group of infants, they were the ones that showed the most remarkable increase of activated memory $T$ cells.

Children with asymptomatic Giardia infection may have an advantage over uninfected children in their nutritional status and in freedom from other illnesses (2). In the present study, lymphocyte subsets of infants with diarrhea infected with $G$. lamblia resembled those observed in control infants without diarrhea. In particular, infection with $G$. lamblia, as opposed to other causes of acute gastroenteritis, was not accompanied by an increase in memory CD4+ cells. This phenomenon may be due to the fact that this parasite remains confined to the intestinal lumen without invading the intestinal mucosa. It may thereby fail to elicit either a systemic inflammatory response or alterations in lymphocyte subsets in the peripheral blood. The small bowel morphologic changes observed during $G$. lamblia infection are believed to be determined, at least in part, by $\mathrm{T}$ celldependent activity (25). Indeed, it is the gut-associated lymphoid tissue that plays a predominant role in the host defense against intestinal pathogens. T cells expressing mainly the CD4 antigen are present in the lamina propria, whereas $T$ cells expressing the CD8 antigen and NK cells compose the majority of the intraepithelial lymphocytes $(26,27)$. To what degree the local intestinal immune response and changes in intestinal lymphocyte subsets are in fact mirrored by those detected in the peripheral blood remains to be determined. In AIDS patients, the decrease in the proportion of helper $\mathrm{T}$ cells and increase in cytotoxic-suppressor $T$ cells observed in the peripheral blood is similarly noted when studying the lymphocyte population of their intestinal mucosa (28). Further studies are therefore needed to elucidate the changes in intestinal mucosal lymphocyte subsets occurring during $G$. lamblia infection and to correlate them with lymphocyte subset distribution in the circulation.

It could be argued that, in the absence of more extensive viral studies, the mere presence of $G$. lamblia trophozoites and cysts in the stool may not be sufficient proof that the parasite is the cause of the diarrheal disease. If indeed the diarrhea in Giardiapositive cases was caused by another concomitant pathogen, then our findings may suggest that $G$. lamblia infection imparts an inhibitory effect on the cellular immune response, abrogating the expected increase in the level of helper-inducer or memory CD4+ cells as observed among the acute gastroenteritis group.

Our observation that, in contrast to the dramatic changes in PBL subsets in infants with acute gastroenteritis, G. lambliaassociated diarrhea is not accompanied by similar changes is intriguing and warrants further study of the immune response elicited by these pathogens.

Acknowledgments. The authors thank Yael Keren-Zur, Chaviva Wiener, and Becky Huri for their technical assistance.

\section{REFERENCES}

1. Kay R, Barnes GL, Townley RRW 1977 Giardia lamblia infestation in 154 children. Aust Paediatr J 13:98-104

2. Ish-Horowicz M, Korman S, Shapiro M, Har-even U, Tamir I, Strauss N, Deckelbaum RJ 1988 Asymptomatic giardiasis in children. Pediatr Infect Dis J 8:773-779

3. Ament ME, Rubin CE 1972 Relation of giardiasis to abnormal intestina structure and function in gastrointestinal immunodeficiency syndromes. Gastroenterology 62:216-226

4. Korman S, Granot E, Ramu N 1989 Severe giardiasis in a child during cancer therapy. Am J Gastroenterol 84:450-451

5. Kumkum Khanna R, Nain CK, Mehta S, Vinayak VK 1988 Depressed humoral immune responses to surface antigens of Giardia lamblia in persistent giardiasis. Pediatr Infect Dis J 7:492-498

6. John Goka AK, Mathan VI, Rolston DDK, Farthing MJG 1986 Diagnosis of giardiasis by specific IgM antibody enzyme linked immunosorbent assay. Lancet 2:184-186

7. Birkhead G, Janoff EN, Vogt RL, Smith PD 1989 Elevated levels of immunoglobulin A to Giardia lamblia during a waterborne outbreak of gastroenteritis. J Clin Microbiol 27:1707-1710

8. Smith PD, Gillin FD, Spira WH, Nash TE 1982 Chronic giardiasis: studies on drug sensitivity, toxin production and host immune response. Gastroenterology 83:797-803

9. Krensky AM, Lanier LL, Engelman EG 1985 Lymphocyte subsets and surface molecules in man. Clin Immunol Rev 4:95-138

10. Horejsi V 1991 Surface antigens of human leukocytes. Adv Immunol 49:75147

11. Abo T, Balch CM 1981 A differentiation antigen of human NK and K cells identified by a monoclonal antibody (HNK-1). J Immunol 127:1024-1029

12. Stites DP, Casavant CH, McHugh TM, Moss AR, Beal SL, Ziegler JL, Saunders AM, Warner NL 1986 Flow cytometric analysis of lymphocyte phenotypes in AIDS using monoclonal antibodies and simultaneous dual immunofluorescence. Clin Immunol Immunopathol 38:161-177

13. Sanders ME, Makgoba MW, Shaw S 1988 Human naive and memory T-cells: reinterpretation and further characterization of helper-inducer and suppressor-inducer subsets. Immunol Today 9:195-199

14. Beverley PCL, Merkenschlager M, Wallace DL 1989 Identification of human naive and memory $T$ cells. In: Melchers $F$ (eds) Progress in Immunology, Vol 7. Springer Verlag, Berlin, pp 432-438

15. Pirruccello SJ, Collins M, Wilson JE, McManus BM 1989 Age-related changes in naive and memory CD4 $\mathrm{T}$ cells in healthy human children. Clin Immunol Immunopathol 52:341-345

16. Hoffman RA, Kung PC, Hansen WP, Goldstein G 1980 Simple and rapid measurement of human T-lymphocytes and their subclasses in peripheral blood. Proc Natl Acad Sci USA 77:4914-4917

17. Packs DR, Herzenberg LA: 1984 Fluorescence-activated cell sorting: theory, experimental optimization and applications in lymphoid cell biology. Methods Enzymol 108:197-241

18. Morimoto C, Letvin NL, Distaso JA, Aldrich WA, Schlossman SF 1985 The isolation and characterization of the human suppressor-inducer $\mathrm{T}$ cell subset. J Immunol 134:1508-1515

19. Morimoto C, Letvin NL, Boyd AW, Hagman M, Brown HM, Kornacli MH Schlossman SF 1985 The isolation and characterization of the human helperinducer subset. J Immunol 134:3762-3769

20. Sanders ME, Makgoba MW, Sharrow SO, Stephany D, Springer TA, Young HA, Shaw S 1988 Human memory T lymphocytes express increased levels of three adhesion molecules (LFA-3, CD2 and LFA-1) and three other molecules (UCHL1, CDw29 and Pgp-1) and have enhanced IFN- $\gamma$ production. J Immunol 140:1401-1407

21. Thomkinson BE, Wagner DK, Nelson DL, Sullivan JL 1987 Activated lymphocytes during acute Epstein-Barr virus infection. J Immunol 139:38023807

22. Gupta S 1986 Abnormality of Leu $2+7+$ cells in acquired immunodeficiency syndrome (AIDS), AIDS-related complex and asymptomatic homosexuals. $\mathrm{J}$ Clin Immunol 6:502-509

23. Chandra RK 1986 Nutritional regulation of immunity and infection: from epidemiology to phenomenology to clinical practice. J Pediatr Gastroenterol Nutr 5:844-852

24. Koster FT, Palmer DL, Chakraborty J, Jackson T, Curlin GC 1987 Cellular immune competence and diarrheal morbidity in malnourished Bangladeshi children: a prospective field study. Am J Clin Nutr 46:115-120

25. Roberts-Thomson IC, Mitchell GF 1978 Giardiasis in mice. I. Prolonged infection in certain mouse strains and hypothymic (nude) mice. Gastroenterology 75:42-46

26. Selby WS, Janossy G, Bofill M, Jewell DP 1983 Lymphocyte subpopulations in the human small intestine. The findings in normal mucosa and in the mucosa of patients with adult coeliac disease. Clin Exp Immunol 52:219228

27. Kelly J, O'Farrelly C, O'Mahoney C, Weir DG, Feighery C 1987 Immunoperoxidase demonstration of the cellular composition of the normal and coeliac small bowel. Clin Exp Immunol 68:177-188

28. Budhhraja M, Levendolu H, Kocka F, Mangkornkanock M, Sherer R 1987 Duodenal mucosal $\mathrm{T}$ cell subpopulation and bacterial cultures in acquired immune deficiency syndrome. Am J Gastroenterol 82:427-431 